\title{
Cross-optical-beam nonlinear photoacoustic microscopy
}

Liren Zhu, Liang Gao, Lei Li, Lidai Wang, Teng Ma, et al.

Liren Zhu, Liang Gao, Lei Li, Lidai Wang, Teng Ma, Qifa Zhou, K. Kirk Shung, Lihong V. Wang, "Cross-optical-beam nonlinear photoacoustic microscopy," Proc. SPIE 8943, Photons Plus Ultrasound: Imaging and Sensing 2014, 89433H (3 March 2014); doi: 10.1117/12.2037926

SPIE. Event: SPIE BiOS, 2014, San Francisco, California, United States 


\title{
Cross-optical-beam nonlinear photoacoustic microscopy \\ Liren $\mathrm{Zhu}^{\mathrm{a}}$, Liang $\mathrm{Gao}^{\mathrm{a}}$, Lei Li ${ }^{\mathrm{a}}$, Lidai Wang ${ }^{\mathrm{a}}$, Teng Ma ${ }^{\mathrm{b}}$, Qifa Zhou ${ }^{\mathrm{b}}, \mathrm{K}$. Kirk Shung ${ }^{\mathrm{b}}$ and Lihong V. Wang*a \\ ${ }^{a}$ Optical Imaging Laboratory, Department of Biomedical Engineering, Washington University, St. Louis, MO 63130; ${ }^{b}$ Department of Biomedical Engineering, University of Southern California, Los Angeles, CA 90089
}

\begin{abstract}
We present a photoacoustic microscopy (PAM) technique with an optical sectioning capability. By combining crossoptical-beam illumination with nonlinear PAM, an axial resolution of $8.7 \mu \mathrm{m}$ was measured, demonstrating a fourfold improvement over the acoustically determined value. Compared to methods relying on high-frequency ultrasound transducers to improve the axial resolution, our approach offers a greater working distance and a higher signal-to-noise ratio.
\end{abstract}

Keywords: photoacoustic microscopy, optical sectioning, nonlinear optics, axial resolution, cross-beam illumination

\section{INTRODUCTION}

Optical resolution photoacoustic microscopy (OR-PAM) has demonstrated great potential in functional and molecular imaging both in vivo and in vitro ${ }^{1,2}$, due to its high spatial resolution. In most of the current OR-PAM systems, optical diffraction-limited lateral resolution has been achieved up to the sub-micron level ${ }^{3}$. However, they rely on time-resolved acoustic detection to provide an axial resolution of around tens of microns. In the past decade, many strategies have been proposed to enhance the axial resolution of OR-PAM systems. The predominant approach is to increase the bandwidth of detected acoustic signals by using high frequency, broadband sensors ${ }^{4,5,6}$. However, due to the severe attenuation that high frequency acoustic waves experience in biological tissue, the use of broadband sensors usually results in low signal-tonoise ratio (SNR). Additionally, broadband sensors usually suffer from a limited working distance, which makes these techniques harder to implement in reflection mode systems. Recently, optical sectioning has been demonstrated in PAM through the use of nonlinear photoacoustic signals ${ }^{7}$. In these methods, optical nonlinearity is used to confine the generation of nonlinear PA signals within a small and optically defined volume. Similar to optical sectioning techniques in other optical microscopy, the axial resolution generated by these methods depends on the numerical aperture (NA) of the optical system. In general, higher NA is required to provide an axial resolution better than the acoustically defined value in conventional OR-PAM. However, the use of high-NA objectives reduces the system's flexibility for in vivo imaging. To overcome these limitations, we developed an optical sectioning OR-PAM by integrating cross-beam illumination with nonlinear photoacoustic effects. By employing cross-beam illumination with two low NA objectives, we created an optically-confined excitation volume. Furthermore, by leveraging nonlinear effects such as absorption saturation and nonlinear thermal expansion, we extracted signals associated with higher-order fluence distribution. Since low-NA objectives and ultrasound transducers with relatively low bandwidth are allowed, the system's working distance reaches the centimeter scale.

\section{THEORY AND MODELING}

When optically absorbing materials, including biological tissue, are pumped by sufficient light energy, the amplitude of the generated PA signal becomes nonlinearly dependent on the input energy. The major sources of such photoacoustic nonlinearity include absorption saturation ${ }^{8}$ and temperature dependence of the thermal expansion coefficient ${ }^{9}$. Such a nonlinear relationship between PA amplitude and input energy carries information that is related to higher-order optical fluence distribution. To extract this information and form an image with a better resolution, multiple A-lines are acquired at each scanning position with different pulse energies. A curve of pulse energy vs. PA amplitude is then formed and fitted to a $3^{\text {rd }}$ order polynomial model, and the $3^{\text {rd }}$ order coefficient of the fitted model is used as the value of this voxel. By modeling these two major resources of nonlinearity, the $n$-th order coefficient extracted from the described procedure is formed as:

Photons Plus Ultrasound: Imaging and Sensing 2014, edited by Alexander A. Oraevsky, Lihong V. Wang, Proc. of SPIE Vol. 8943, 89433H · (c) 2014 SPIE · CCC code: 1605-7422/14/\$18 · doi: 10.1117/12.2037926 

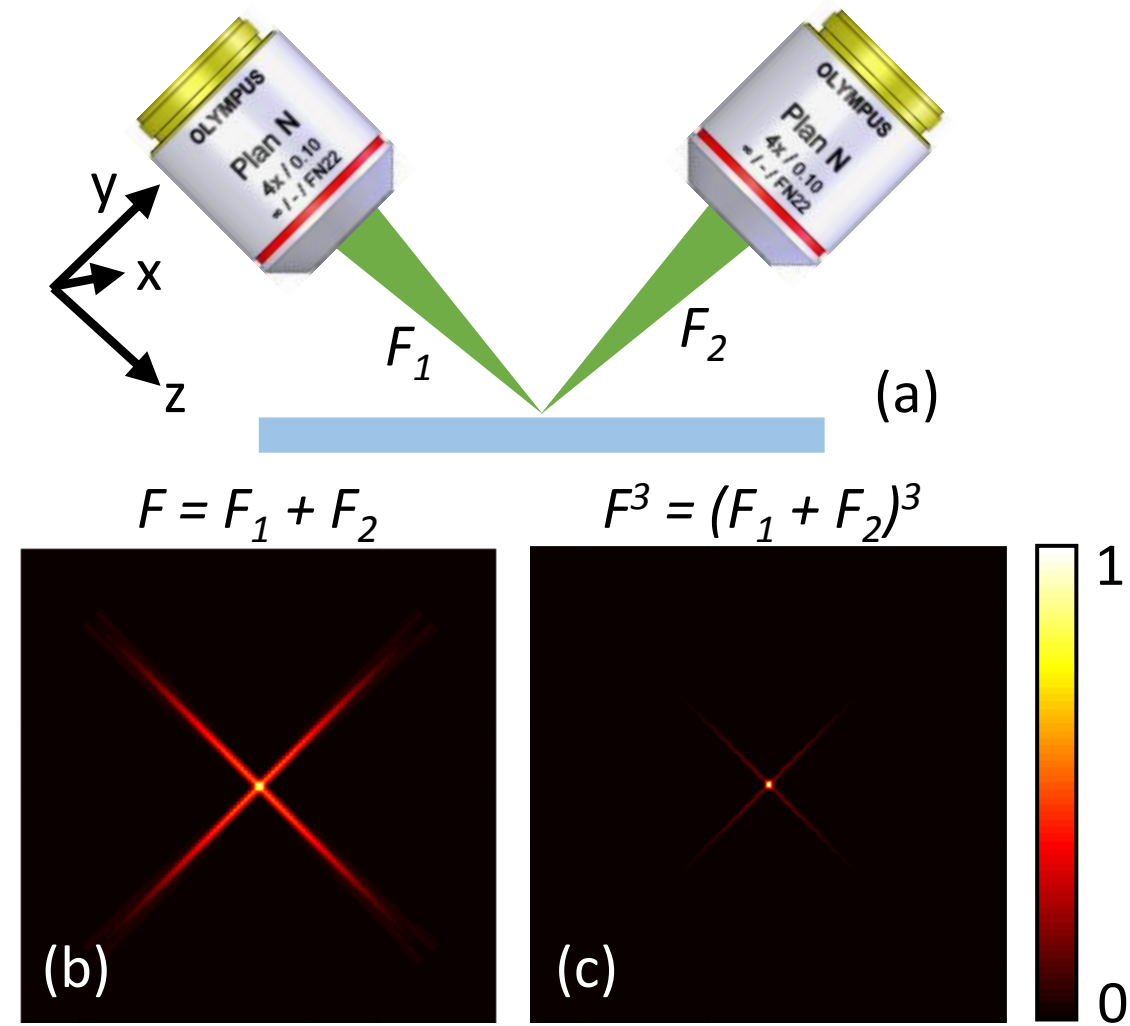

Figure 1 Illustration of the general idea of cross-beam nonlinear OR-PAM.

$$
c_{n}\left(\mathbf{r}_{0}\right)=(-1)^{n-1} \cdot \int_{\mathbf{r}}\left[A_{1} \cdot \mu_{a 0}(\mathbf{r})-A_{2} \cdot \frac{n-1}{2} \cdot \mu_{a 0}^{2}(\mathbf{r})\right] \cdot\left(\hat{F}\left(\mathbf{r}-\mathbf{r}_{0}\right)\right)^{n} \cdot \mathrm{d} \mathbf{r},
$$

where $A_{1}, A_{2}$ are constants, $\mu_{a 0}$ is the unsaturated absorption coefficient, and $\hat{F}$ is the optical fluence distribution of the focusing system. This analysis predicts that the spatial resolution of the $\mathrm{n}$-th order image is determined by the $\mathrm{n}$-th order fluence distribution.

In theory, a direct implementation of this technique can equip a conventional, single-illumination OR-PAM system with optical sectioning capability. However, when low-NA objectives are used, the axial resolution provided by this optical sectioning capability is not ideal. Take our experimental setup (fully described later) for example. With an optical NA of 0.1 and $532 \mathrm{~nm}$ wavelength, the full width at half maximum (FWHM) of the linear fluence distribution along the axial direction is approximately $95.8 \mu \mathrm{m}$. If the $3^{\text {rd }}$ order coefficient is extracted, the improved axial resolution is $55.3 \mu \mathrm{m}$, which is worse than the typical acoustically determined value.

Our idea is to combine photoacoustic nonlinearity with cross-beam illumination in low-NA objective based OR-PAM, in order to enhance axial resolution while maintain a relatively long working distance. Cross-beam illumination will create an optically confined, high intensity confocal area, as shown in Figure 1(b). By extracting the $3^{\text {rd }}$ order coefficient, the response from outside of the confocal area is further suppressed, as shown in Figure 1(c). To demonstrate the feasibility of this idea, we modelled the point spread function (PSF) of the proposed method. Modeling was done using the Fresnel diffraction theory in a clear medium ${ }^{10}$. First, the optical fluence distribution produced by a thin, ideal lens with a circular aperture and 0.1 NA was calculated at each plane perpendicular to the lens' optical axis, around the focal point. The single illumination PSF was obtained by taking the cube of this distribution (see Figure 2, blue, dashed curve). The optical fluence distribution produced by cross-beam illumination was calculated by adding two single-lens fluence distribution together. The corresponding PSF was obtained again by taking the cube of this distribution (see Figure 2, red, solid curve). Along the optical axes of both lenses, the cross-beam PSF has a FWHM of $2.5 \mu \mathrm{m}$, while single illumination PSF has a FWHM of $57.5 \mu \mathrm{m}$. In the lateral direction, on the other hand, both PSFs have the same shape and same FWHM of $1.5 \mu \mathrm{m}$. 

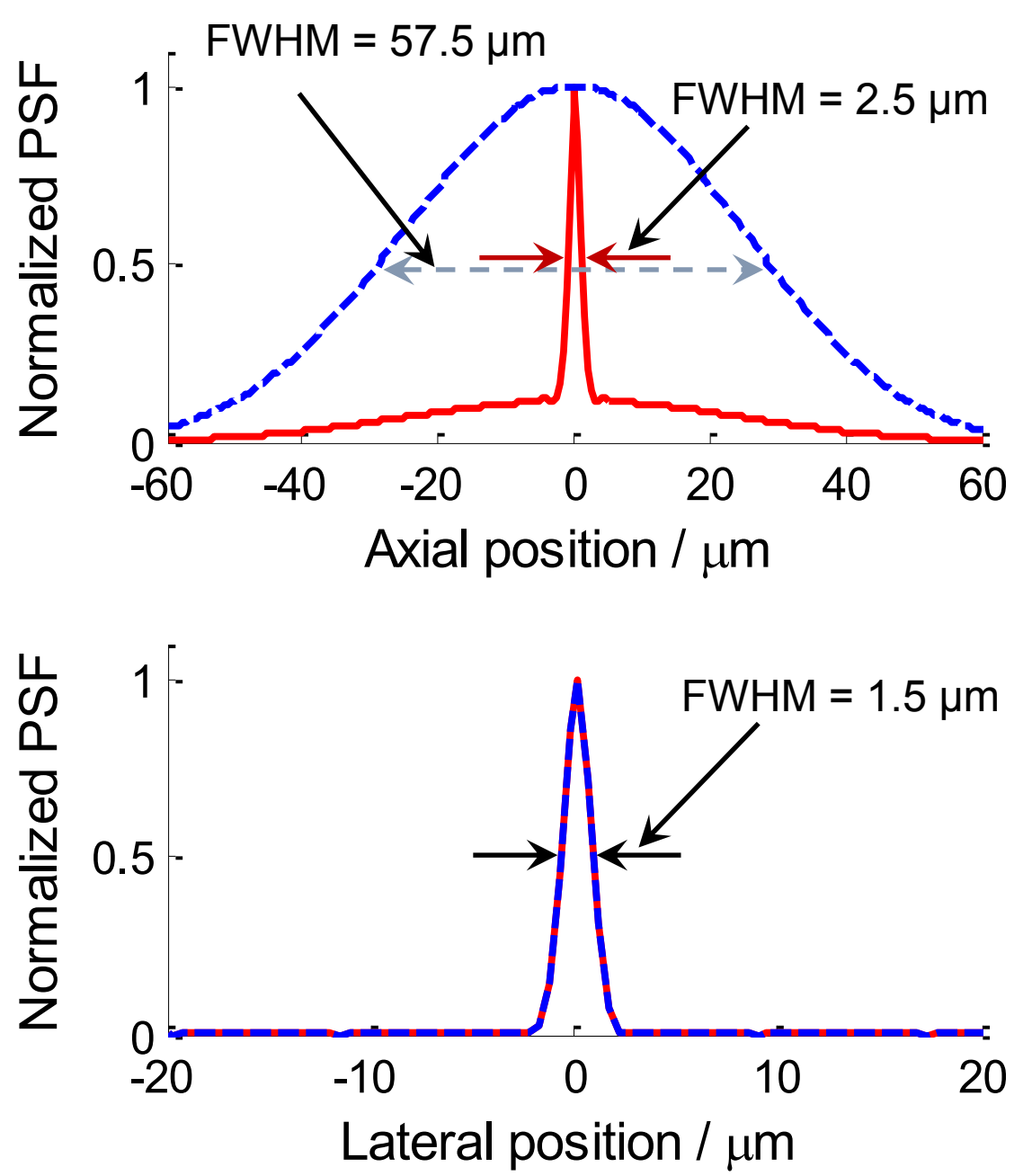

Figure 2 Modeling results: third order theoretical lateral and axial PSFs of single illumination (blue) and cross-beam illumination (red), obtained using diffraction theory and two 0.1 NA ideal lenses.

\section{EXPERIMENTS AND RESULTS}

Figure 1 shows the experimental setup. The system employs an Nd: YVO4 laser (AOT-YVO-100Q, AOT Inc.) as the light source (wavelength $532 \mathrm{~nm}$ ). A variable neutral density (ND) filter (NDC-50C-2M, Thorlabs) is placed right after the laser output and adjusted by a stepper motor to vary the pulse energy. The output laser beam ( $8 \mathrm{kHz}$ repetition rate, $2 \mathrm{~ns}$ pulse width) is then sent through a spatial filter consisting of two lenses (LA1131, Thorlabs) and a 50- $\mu \mathrm{m}$ pinhole (P50C, Thorlabs). The pulse energy is monitored by a photodiode (PD) sensor. Then, the laser beam is split into 2 beams using a polarization-based variable beam splitter. Each of these two beams is directed through an objective lens (UIS2 PLN4X, NA of 0.1 , Olympus) and focused on the sample, both vertically from the bottom and horizontally from the side. The side illumination is deferred by a delay line consisting of two mirrors and a corner mirror, so that the time delay can be adjusted to make the temporal profile of the two illuminations overlap at the sample. The sample is mounted on a cover glass and immersed in water. Acoustic waves are detected from the top. We also built a custom-made focused ultrasonic transducer (central frequency $\sim 48 \mathrm{MHz}$, bandwidth $\sim 38 \mathrm{MHz}$ ), with long working distance $(11 \mathrm{~mm}$ ), to persist our overall working distance at the centimeter level. 
We quantified the resolution along both axial and lateral directions by imaging a thin layer of ink. We first moved the sample to the confocal spot and scanned it in one direction. The axial PSF is interpolated under the assumption of symmetry. The lateral resolution was quantified by imaging a thin ink layer with a sharp edge.

The results from the axial resolution measurement were interpolated to a cubic spline because the shape of the axial point spread function (PSF) is not Gaussian. The full width at half maximum (FWHM) of the interpolated curve is $8.68 \mu \mathrm{m}$. In the lateral resolution measurement, an edge spread function (ESF) was obtained. The data were fitted to an error function model, and the FWHM of the corresponding Gaussian PSF is $5.14 \mu \mathrm{m}$.

On the other hand, the acoustically determined axial resolution (FWHM) can be estimated by this formula ${ }^{4}, 0.88 v_{a} / B W_{a}$, where $v_{a}$ is the speed of sound in the surrounding medium and $B W_{a}$ is the bandwidth of acoustic detection. In our experimental setup, the acoustically determined value is $34.74 \mu \mathrm{m}$. Therefore, the measured resolution $8.68 \mu \mathrm{m}$ is a fourfold improvement over the acoustically determined axial resolution.

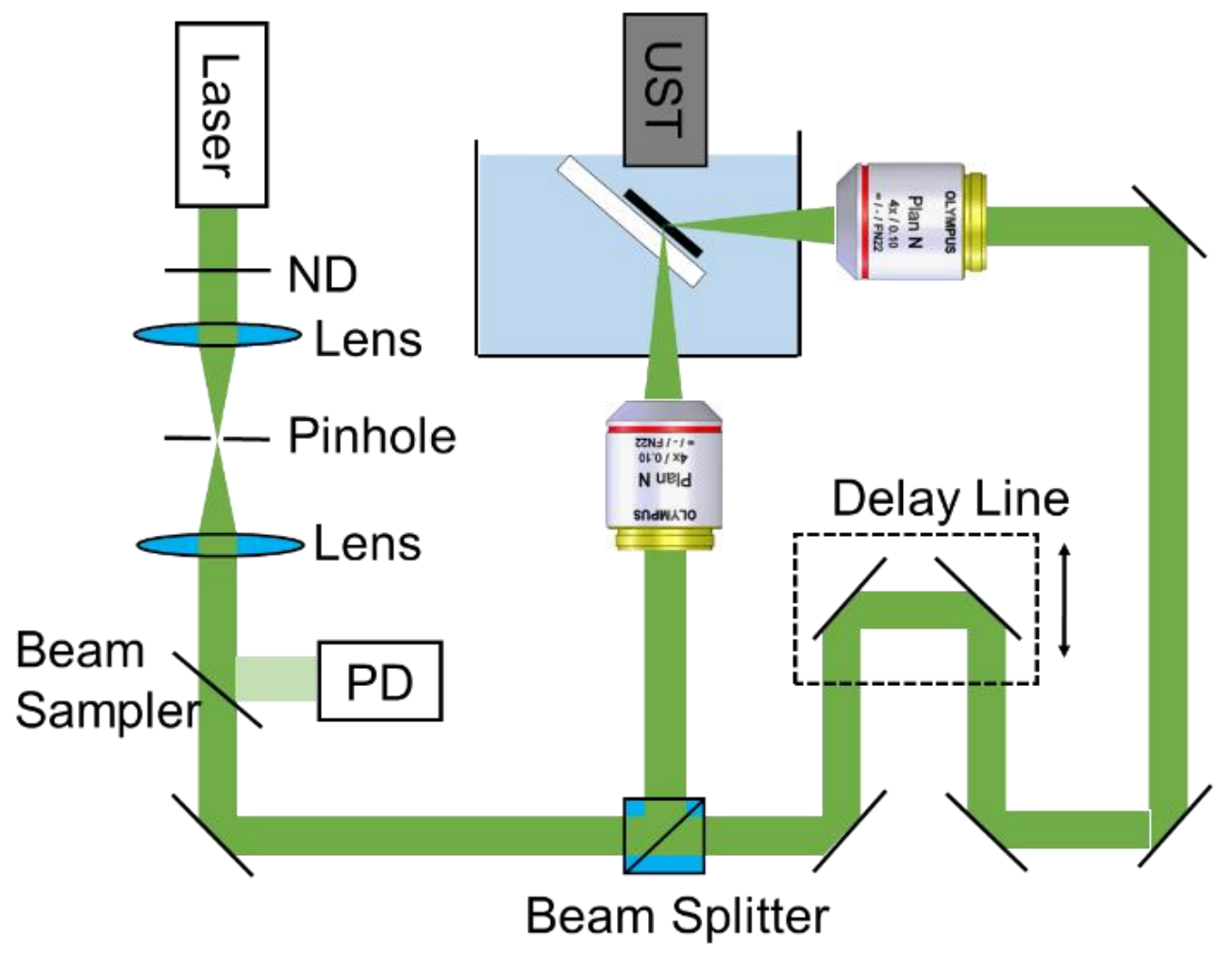

Figure 3 Experimental setup of cross-beam nonlinear photoacoustic microscopy.

\section{CONCLUSION}

As we demonstrated in our modeling results, the theoretical limit of axial resolution, when 3rd order component is extracted, is $2.5 \mu \mathrm{m}$, which is better than the $8.7 \mu \mathrm{m}$ that we achieved experimentally. This degradation of spatial resolutions is due to the imperfect alignment of the two illumination beams. If better alignment of the two beams can be achieved, using custom-made mounting for example, even better axial resolution is possible.

In conclusion, we have demonstrated the feasibility of achieving optical sectioning using cross-beam nonlinear PAM. The resultant axial resolution is $8.7 \mu \mathrm{m}$, a fourfold improvement over the acoustically determined value. Compared to existing high-frequency-transducer-based methods, our method has a centimeter-scale working distance and is not subject to severe attenuation of high-frequency PA waves in biological tissue. 

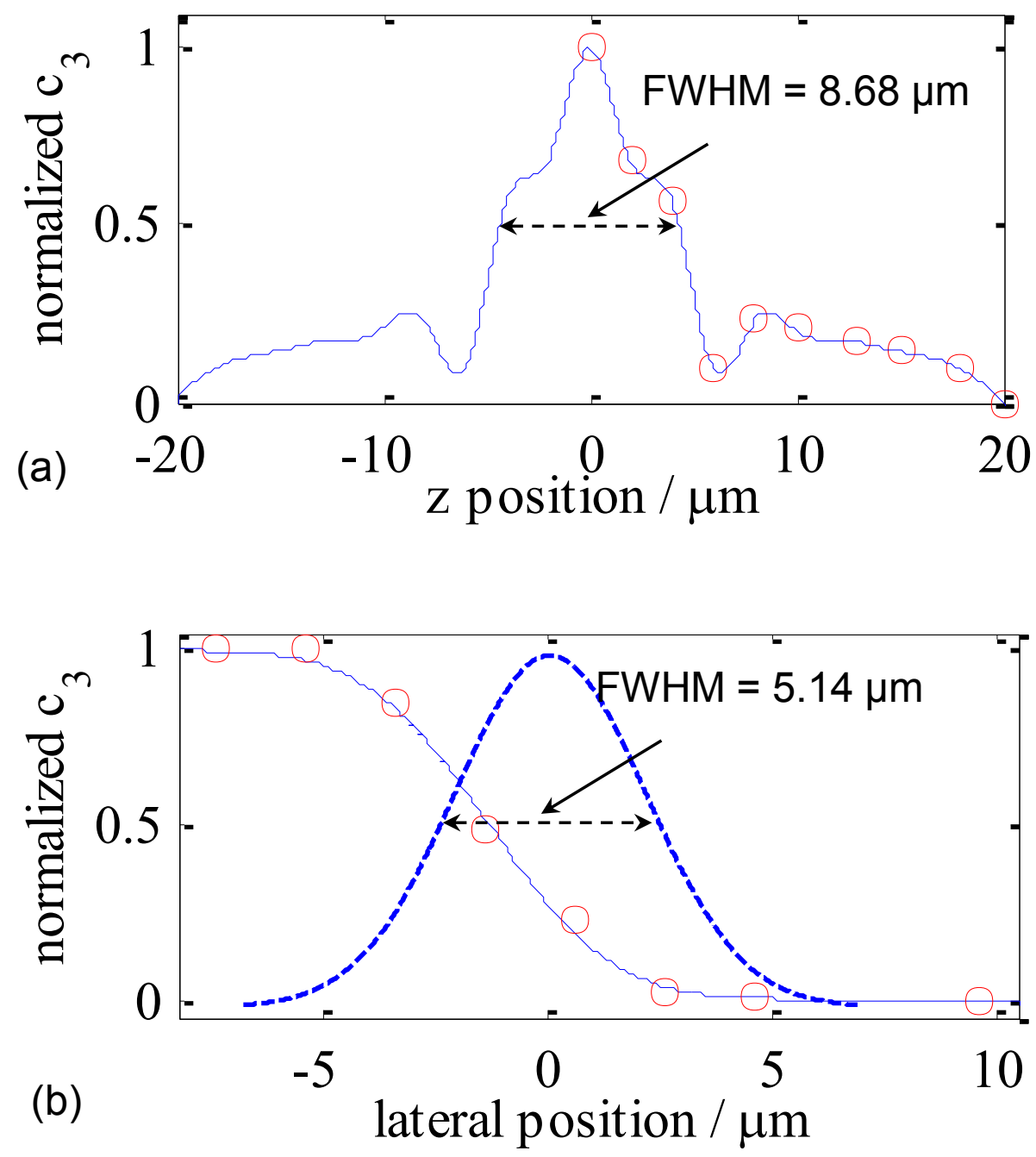

Figure 4 Experimental quantification of axial (a) and lateral (b) resolution. Red circles are the measured data and blue curves are the interpolated or fitted PSF and ESF.

\section{REFERENCES}

[1] Wang, L.V. and Hu, S., "Photoacoustic Tomography: In Vivo Imaging from Organelles to Organs," Science 335, 1458-1462 (2012).

[2] Hu, S. and Wang, L.V., "Optical-Resolution Photoacoustic Microscopy: Auscultation of Biological Systems at the Cellular Level," Biophysical Journal 105(4), 841-847 (2013).

[3] Zhang, C., Maslov, K., Hu, S., Chen, R., Zhou, Q., Shung, K.K. and Wang, L.V., "Reflection-mode submicronresolution in vivo photoacoustic microscopy." Journal of Biomedical Optics 17(2), 020501 (2012).

[4] Zhang, C., Maslov, K., Yao, J. and Wang, L.V., "In vivo photoacoustic microscopy with 7.6- $\mu \mathrm{m}$ axial resolution using a commercial 125-MHz ultrasonic transducer," Journal of Biomedical Optics 17(11), 116016 (2012).

[5] Zhang, C., Zhou, Y., Li, C., Wang, L.V., "Slow-sound photoacoustic microscopy," Applied Physics Letters 102(16), 163702 (2013).

[6] Xie, Z., Chen, S. L., Ling, T., Guo, L. J., Carson, P. L. and Wang, X., "Pure optical photoacoustic microscopy," Optics express 19(10), 9027-9034 (2011).

[7] Shelton, R. L., Mattison, S. P. and Applegate, B. E., "Volumetric imaging of erythrocytes using label-free multiphoton photoacoustic microscopy," Journal of biophotonics 7(1), (2013).

[8] Siegman, A.E., [Lasers], University Science Books, (1986).

[9] Calasso, I. G., Craig, W. and Diebold, G. J., "Photoacoustic point source," Phys. Rev. Lett. 86, 3550-3553 (2001).

[10] Barrett, H. H. and Myers, K. J., [Foundations of Imaging Science], Wiley-VCH, (2003). 